\title{
Lapatinib protects against epileptic seizures via halting glutathione peroxidase 4-dependent ferroptosis
}

\author{
Jining Jia ${ }^{1}$, Qin $\mathrm{Li}^{1}$, Qianyi Sun ${ }^{1}$, Nan Yang${ }^{1}$, Kangni Chen ${ }^{1}$, Xixi Yin ${ }^{1}$, Hong-Hao Zhou ${ }^{2}$, \\ and Xiaoyuan $\mathrm{Mao}^{1}$ \\ ${ }^{1}$ Xiangya Hospital Central South University \\ ${ }^{2}$ Department of Clinical Pharmacology, Xiangya Hospital, Central South \\ University;Institute of Clinical Pharmacology, Central South University; Hunan Key \\ Laboratory of Pharmacogenetics
}

May 19, 2020

\begin{abstract}
Background and Purpose: Repetitive epileptic seizures trigger massive neuronal death. Therefore, neuroprotection plays a role in preventing neuronal death and inversely suppresses seizure generation. Additionally, some studies have shown ferroptosis, featured by lipid peroxidation (a dominant form of oxidative stress in the brain), is of paramount importance in epileptic seizures. Lapatinib can play a first-line anti-tumor role by targeting oxidative stress and a recent work illustrates the improvement of encephalomyelitis in rodent models after lapatinib treatment. We hypothesize whether lapatinib can protect against ferroptosis in epileptic seizures via regulating lipid peroxidation. Experimental Approach: The epileptic behavior of the mice was recorded after intracranial injection of KA. Western blot and RT-qPCR were used to detect the protein expression of 4-hydroxynonenal (4-HNE) and glutathione peroxidase 4 (GPX4) and the mRNA expression of prostaglandin endoperoxide synthase 2 (PTGS2) in vivo and in vitro. The level of lipid reactive oxygen species (lipid ROS) in cells pretreated with lapatinib was analyzed by flow cytometry. Key Results: Lapatinib remarkably prevented KA-induced epileptic seizures in mice and ferroptosis was involved in the neuroprotection of lapatinib. Compared with the model group, western blot showed that lapatinib significantly upregulated the levels of GPX4. In the ferroptotic cell death model, lapatinib exerted neuroprotection via up-regulating GPX4. Treatment with Ras-selective lethal small molecule 3 (RSL3), a selective GPX4 inhibitor abrogated its anti-ferroptotic potential. Conclusions and Implications: These results illustrated that lapatinib has neuroprotective potential against KAtriggered epileptic seizures via suppressing GPX4-dependent ferroptosis.
\end{abstract}

\section{Hosted file}

Manuscript.doc available at https://authorea.com/users/323854/articles/452316-lapatinibprotects-against-epileptic-seizures-via-halting-glutathione-peroxidase-4-dependentferroptosis 


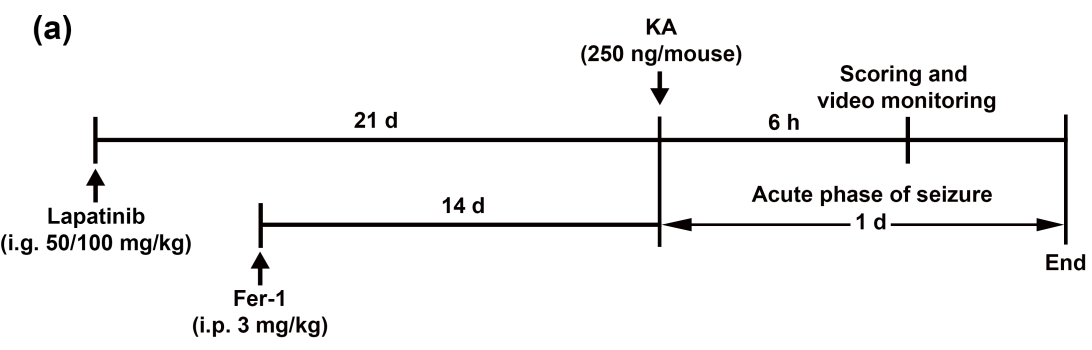

(b)

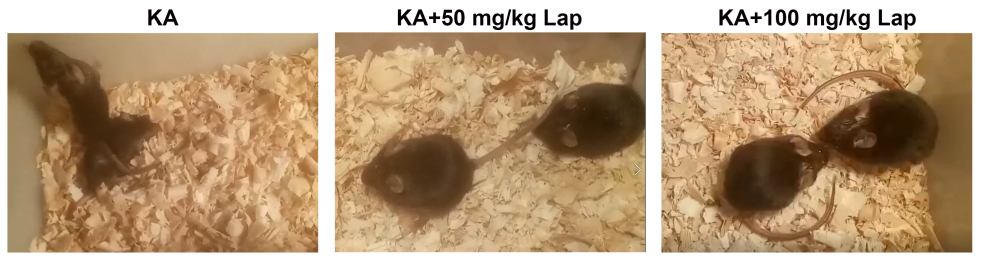

(c)

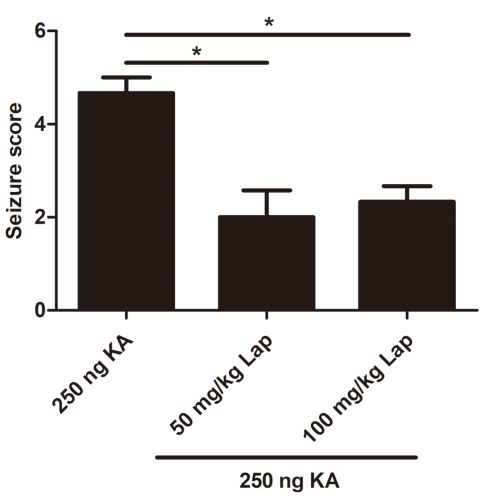

(e)

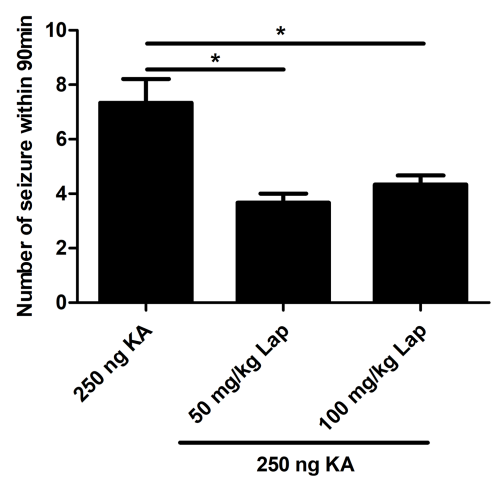

(d)

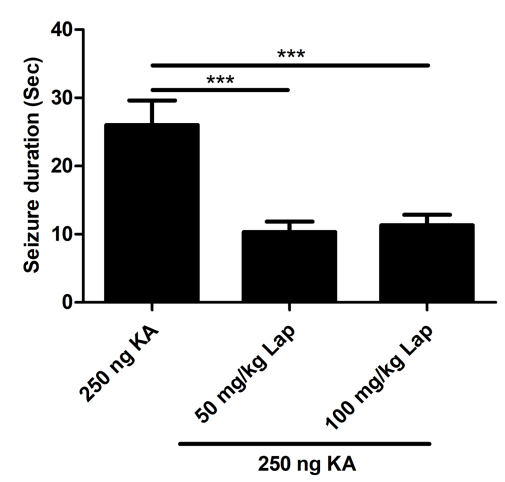

(f)

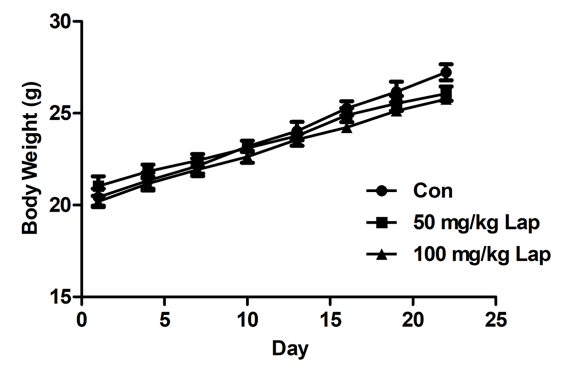


(a)

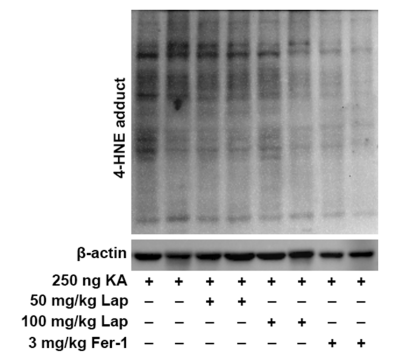

(b)

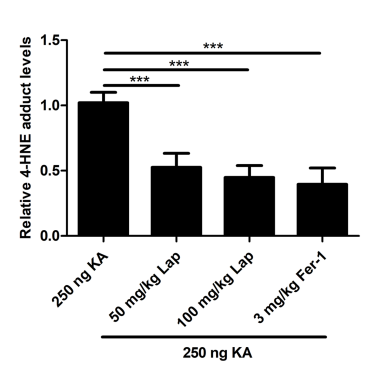

(c)

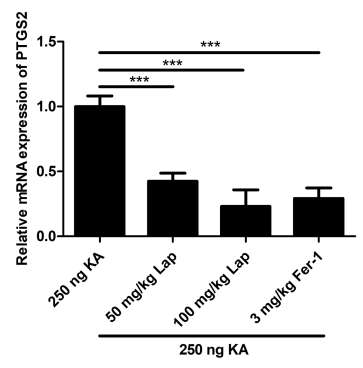


(a)

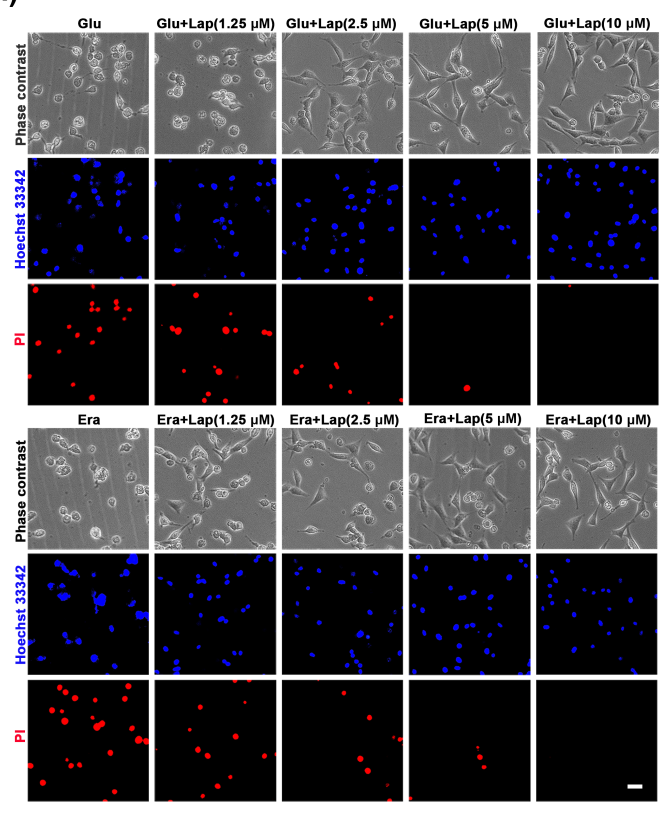

(c)

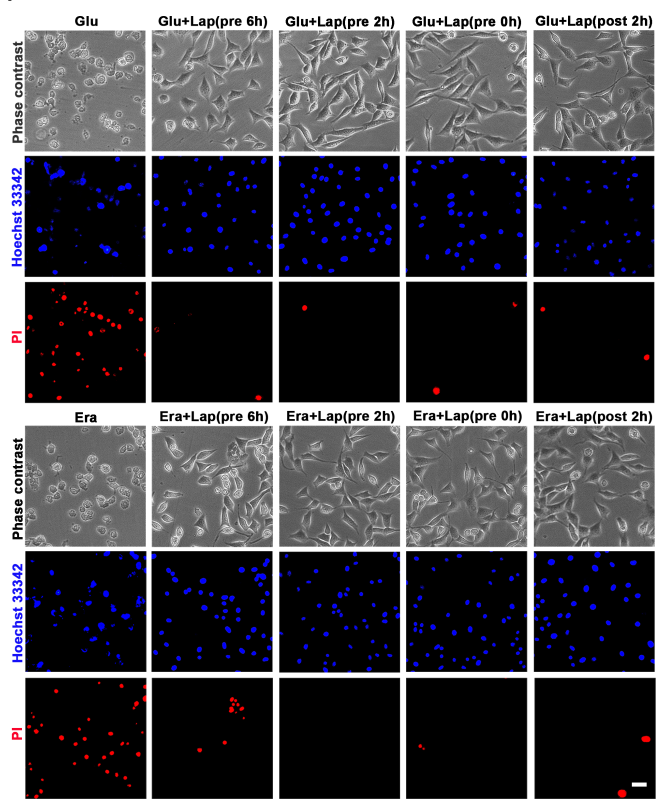

(b)

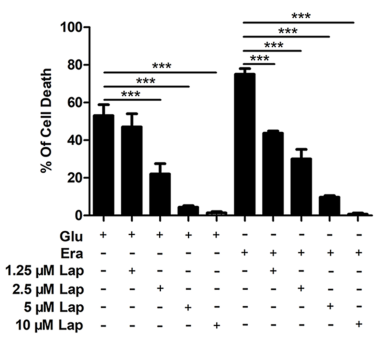

(d)

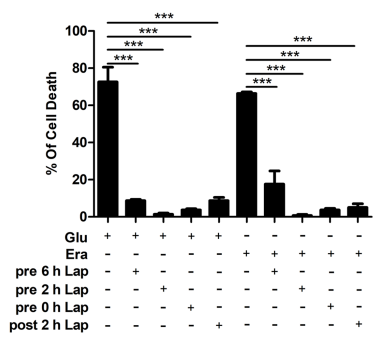

(e)

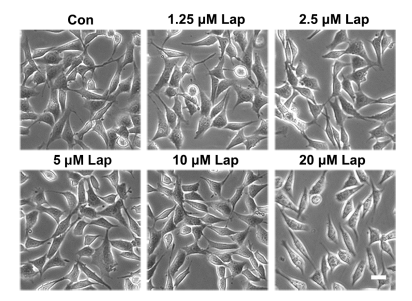


(a)

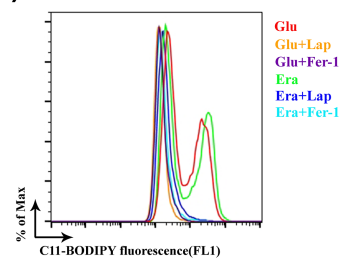

(d)

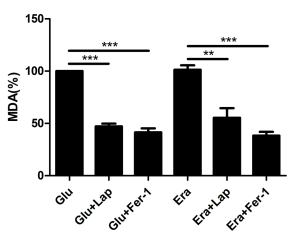

(f)

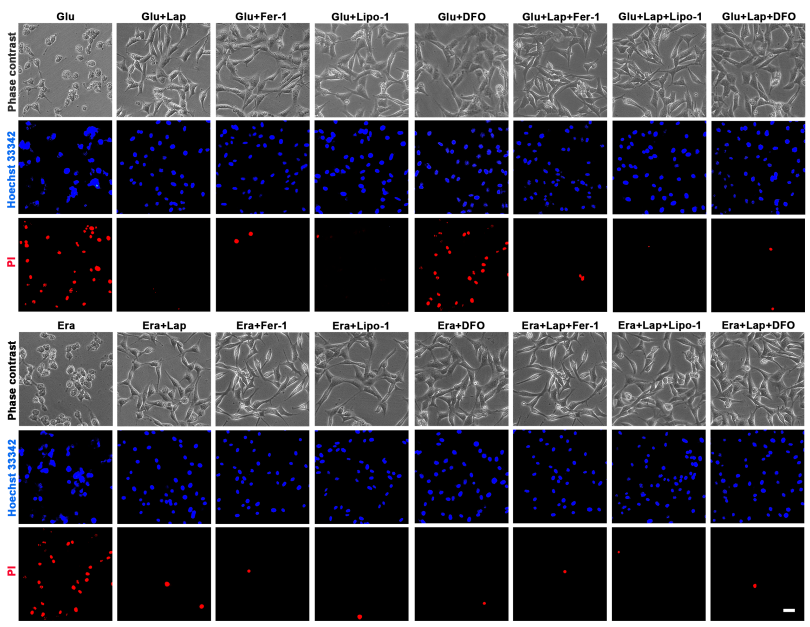

(b)

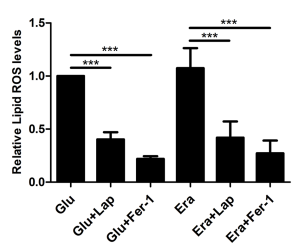

(e)

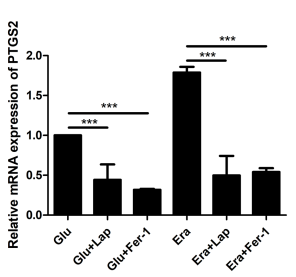

(c)

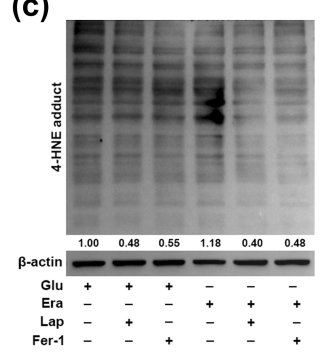

(g)

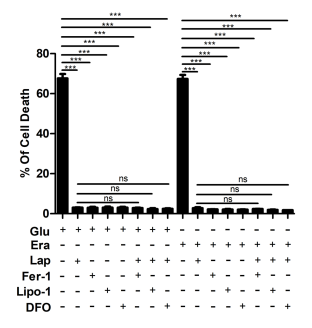


(a)

(b)

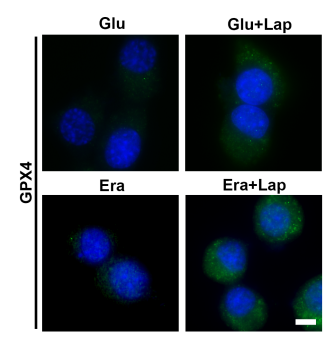

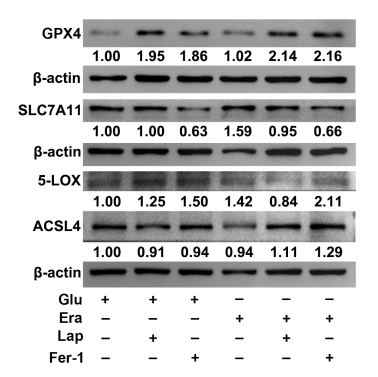

(c)

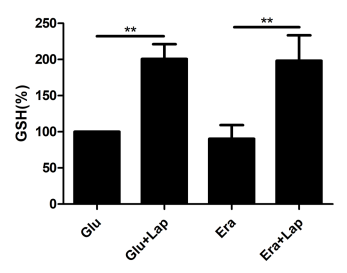

(e)

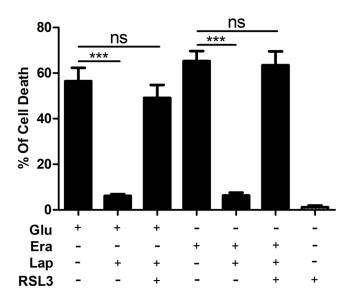

(c)

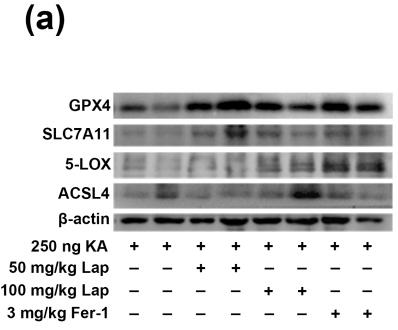

(b)

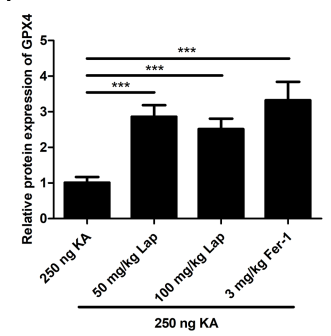

(d)

(e)

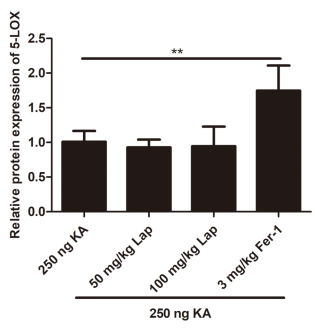

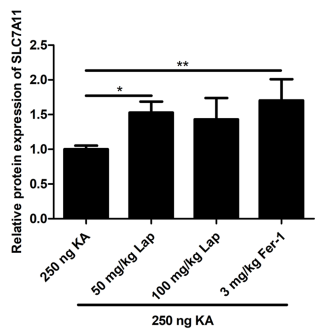

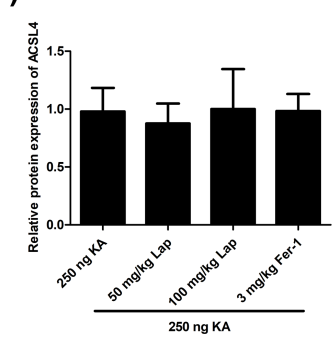


Anti-cancer effect

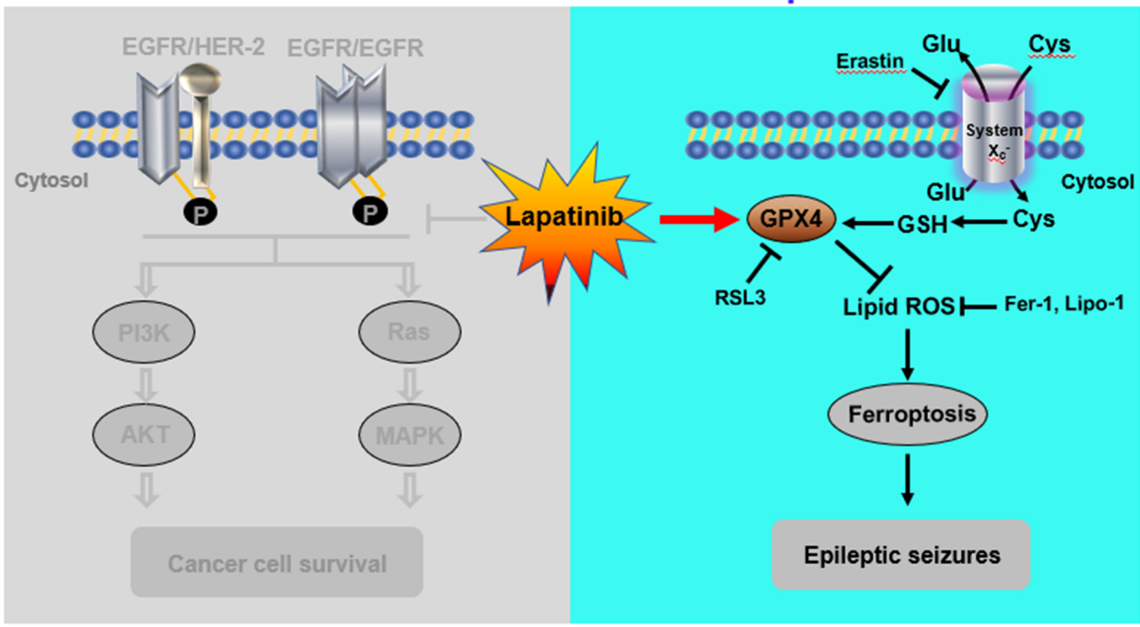

